\title{
Cenários Prospectivos: Uma Visão do Futuro da Presença Feminina em Cursos de Ciência da Computação de uma Instituição de Ensino Superior
}

\author{
Josilene A. Moreira ${ }^{1}$, Ricardo M. Silva ${ }^{2}$, Maria Eulina P. Carvalho ${ }^{3}$ \\ Centro de Informática ${ }^{1}$, Centro de Tecnologia ${ }^{2}$, Centro de Educação ${ }^{3}$ \\ Universidade Federal da Paraíba - UFPB \\ Av. dos Escoteiros - s/n - Distrito Industrial de Mangabeira \\ 58055-000 - João Pessoa-PB \\ josilenedci.ufpb.br ${ }^{1}$, ricardomoreira0203@hotmail.com², \\ mepcarveterra.com.br ${ }^{3}$
}

\begin{abstract}
This article projects scenarios and presents gender equity trend curves using regression analysis, based on historical quantitative series of students in three courses in the Computer Science area of a Brazilian University. In the Bachelor of Science degree in Computer Science it is observed that the number of women is reduced to a rate of $0.4 \%$ per year (aa); without interventions to reverse this trend, there will be no more women in 2050. In Computer Engineering there is a decrease in the entry of women to $0.7 \%$ per year; if the fall continues, there will be no women in 2031. In Computational Mathematics, the scenario is indicative of stagnation; there is a tendency to reduce women by $0.01 \%$ per year.
\end{abstract}

Resumo. Este artigo projeta cenários e apresenta curvas de tendência de equidade de gênero utilizando análise de regressão, baseado em séries históricas de quantitativos de estudantes em três cursos da área de Computação de uma IES do Brasil. No curso de Bacharelado em Ciência da Computação constata-se que o número de mulheres reduz-se a uma taxa de 0,4\% ao ano (aa); sem intervenções para reverter essa tendência, não haverá mais mulheres em 2050. Na Engenharia da Computação há diminuição na entrada de mulheres a 0,7\% aa; caso a queda se mantenha, não haverá mulheres em 2031. Já na Matemática Computacional, o cenário é indicativo de estagnação; há uma tendência de redução das mulheres em 0,01\% ao ano.

\section{Introdução}

De acordo com o Censo da Educação Superior, as mulheres constituíram a maioria dos estudantes matriculados em cursos superiores no Brasil em 2015: do total de 8,3 milhões elas correspondem a 55,6\%. Elas também superam os homens número de ingressantes $(53,9 \%)$ e de concluintes $(59,9 \%)$. Entretanto, quando analisamos os cursos e áreas de ingresso, verificamos uma polarização entre as escolhas de homens e mulheres: elas predominam nas áreas compreendidas como femininas, tipicamente ligadas ao cuidado, enquanto eles predominam na área tecnológica. Entre os dez maiores cursos de graduação em número de matrículas por sexo as mulheres são maioria em Pedagogia, Enfermagem, Psicologia, Serviço Social, Gestão de Pessoas, Fisioterapia e Arquitetura, enquanto os homens são maior número em Engenharia Civil, 
Engenharia Mecânica, Engenharia de Produção, Formação de Professor em Educação Física, Análise e Desenvolvimento de Sistemas e Engenharia Elétrica ${ }^{1}$ (INEP, 2016).

A Universidade Federal da Paraíba (UFPB) foi criada pela Lei Estadual 1.366, de 02/12/1955, sob o nome de Universidade da Paraíba e posteriormente, com a sua federalização, aprovada e promulgada pela Lei $\mathrm{n}^{\circ}$. 3.835 de 13/12/1960, foi transformada em Universidade Federal da Paraíba. Nos últimos cinco anos, com a adesão ao novo Plano de Reestruturação e Expansão das Universidades (REUNI), do Governo Federal, a UFPB conseguiu dobrar de tamanho e, hoje, já é a instituição de ensino superior do Norte e Nordeste do país a oferecer o maior número de vagas no seu processo seletivo (UFPB, 2016). Possui quatro campi, sendo o Campus I, na cidade de João Pessoa o maior, contando com treze centros. O curso de Bacharelado em Ciência da Computação, criado em 1985, vinculado originalmente ao Departamento de Informática do Centro de Ciências Exatas e da Natureza, encontra-se hoje vinculado ao Centro de Informática (CI), criado em 2012. O CI conta com mais dois cursos na área de Computação: Engenharia da Computação, criado em 2011, e Matemática Computacional, criado em 2012.

Embora recomendada pelo Conselho Econômico e Social das Nações Unidas em 1997 (ONU, 2002), a transversalidade de gênero (gender mainstreaming) na Educação anda em passos lentos Brasil. Admitindo que "a educação brasileira ainda não incorporou totalmente o princípio da igualdade de gênero" e que permanece "o sexismo nas escolhas das carreiras acadêmicas" (Brasil, 2013, p.22), o Plano Nacional de Políticas para Mulheres (PNPM) 2013-2015 propõe "promover políticas para a ampliação do acesso e permanência das mulheres no ensino profissional, tecnológico e no ensino superior, com destaque para as áreas científicas e tecnológicas" (p.23). Entre as ações propostas, indicou políticas de ação afirmativa e campanhas para ampliar o número de mulheres em cursos tradicionalmente masculinos (p.26).

Embora nosso Plano Nacional de Educação - PNE 2014-2024 seja omisso no tocante a essas questões, nosso Ministério da Ciência, Tecnologia e Inovação (MCTI), na Estratégia Nacional de Ciência, Tecnologia e Inovação (ENCTI) 2016-2019, destaca a adoção de programas (como outros países já fizeram) para "implantação de uma política de gênero nas instituições científicas" e a "promoção da paridade (...) a fim de reduzir as desigualdades e combater a discriminação" (BRASIL MCTI, 2016, p.55). Focaliza ainda a redução de disparidades no desenvolvimento das carreiras de CT\&I e a transversalidade da abordagem de gênero nas pesquisas, argumentando que "garantir e incentivar a participação plena e efetiva das mulheres nas Ciências e assegurar a igualdade de oportunidades na área de CT\&I" traz "benefícios diretos para a sociedade como um todo" (p.57).

A realidade atual quanto à igualdade de gênero nos cursos do Centro de Informática segue a tendência da predominância masculina nas áreas de tecnologia, conforme o panorama de todo o Brasil. No curso de Bacharelado em Ciência da Computação as mulheres são 10,2\% (39) dos estudantes matriculados; na Engenharia da Computação elas representam 13,8\% (47) e na Matemática Computacional são 21\% (38). Esta pesquisa, com base em dados quantitativos históricos de alunos ingressantes, matriculados e concluintes nestes cursos desde a sua criação, constrói e apresenta as

${ }^{1}$ Administração, Direito e Ciências Contábeis estão entre os dez maiores cursos em ambos os gêneros. 
curvas de tendências que indicam as possibilidades de cenários futuros quanto à perspectiva de equidade de gênero. Conclui que as desigualdades atuais tendem a permanecer e piorar, na ausência de uma política de inclusão de gênero direcionada à transversalidade e ao alcance da paridade entre discentes.

A técnica de construção de cenários, segundo Schwartz (2000) "é uma ferramenta para ajudar a perceber uma visão em longo prazo (com arte e criatividade) combinando com a prática da conversação estratégica, num mundo de grande incerteza política, social, econômica e tecnológica". Nesse sentido o método de construção de cenários pode ser alicerçado em diferentes paradigmas, ora usando modelos puramente matemáticos, colocando o ser humano em um segundo plano, ora com um foco maior neste último. Em contrapartida, compreendendo-se que os cenários podem ser afetados e modificados por fatores externos, apresenta-se aqui o projeto Meninas na Computação e suas estratégias para alterar a realidade atual e vindoura, promovendo assim uma maior inserção feminina na Ciência da Computação e no eixo da tecnologia.

Espera-se contribuir para o contexto do Ensino Superior em Computação apresentando a realidade da (des)igualdade de gênero e a necessidade de novas abordagens e políticas públicas que possibilitem às mulheres ingressarem em áreas tradicionalmente consideradas masculinas na Ciência e Tecnologia, especificamente na Computação, a partir de um estudo de caso realizado na IFES objeto do nosso estudo.

\section{Inclusão da Perspectiva de Gênero na Educação Superior e na Tecnologia}

Conforme Carvalho, Moreira e Silva (2018), as políticas supranacionais têm enfatizado a promoção do acesso das mulheres às TIC (Tecnologias de Informação e Comunicação), o estímulo para as meninas estudarem STEM (do inglês Science, Technology, Engineering, and Mathematics - Ciências, Tecnologia, Engenharia e Matemática), a necessidade de promover a participação e o avanço das mulheres nos setores tecnológicos, e a mudança dos estereótipos de gênero para que homens e mulheres compartilhem o trabalho doméstico. Dentre os Objetivos de Desenvolvimento Sustentável - ODS Agenda 2030, proclamados pela ONU em 2015, o ODS 5 "Alcançar a igualdade de gênero e empoderar todas as mulheres e meninas" invoca o fim de todas formas de discriminação e a garantia de participação plena e efetiva das mulheres na vida política, econômica e pública (ONU, 2002; ONU, 2015).

A inclusão da perspectiva de gênero na educação superior pode assumir distintos enfoques: institucional, com garantia de apoio à necessária capacitação docente; curricular, envolvendo a transformação da prática docente em conteúdos e metodologia; e comportamental. Sua implementação tem se dado segundo diversas modalidades: transversalidade (a mais ampla e profunda), disciplinas, cursos específicos e eventos. No Brasil tem se dado limitadamente a partir de iniciativas individuais de docentes para a inclusão de conteúdos em disciplinas e oferta de disciplinas (em geral optativas) em alguns cursos, sobretudo nas áreas de ciências sociais e humanas, e mais na pósgraduação do que na graduação. Portanto, a inclusão de gênero na educação superior brasileira padece, em geral, de fraca institucionalização e não alcança a área de STEM (Lima e Costa, 2016).

De fato, as mulheres conquistaram alguns direitos civis, sociais e políticos antes nominalmente próprios dos homens. Entretanto existem algumas permanências, repaginando-se na antiga divisão sexual que estabeleceu espaços, atividades e valores 
distintos e assimétricos para homens e mulheres. Um dos aspectos que demonstram a perpetuação dessa divisão são as escolhas de cursos das áreas em Instituições de Ensino Superior. Nesses cursos há uma relação desigual, e assim, uma aceitação e adesão inconsciente das regras pelos estudantes graças ao "habitus", conforme reforça Bourdieu (1999).

A questão "por que tão poucas?" tem sido feita por estudiosos e educadores, interessados em STEM, em vários países. As respostas envolvem desde a socialização primária e secundária, que reproduz estereótipos de gênero negativos sobre $\mathrm{o}$ desempenho intelectual das mulheres, rebaixando sua autoestima, autoconfiança e autoeficácia, até o clima frio ou hostil nos ambientes acadêmicos (Cooper \& Eddy et al, 2010), além da persistência de crenças sexistas sobre a preponderância do talento inato sobre a aquisição de competências matemáticas e espaciais (Hill, Corbett, St. Rose, 2010). Esses fatores não apenas desviam as meninas da escolha de carreiras em STEM, ao final do ensino secundário ou médio, mas condicionam sua evasão quando ingressam em cursos superiores da área (Blickenstaff, 2005).

Desde a década de 1990, há países que desenvolvem iniciativas para incentivar a inclusão de meninas e mulheres em cursos e carreiras de STEM. Na União Europeia (UE), a política de promoção da equidade de gênero em universidades e institutos de pesquisa tem assumido três abordagens: tratamento igual, ação afirmativa $\mathrm{e}$ transversalidade de gênero. Todavia, o avanço das mulheres nos campos científicos masculinos tem sido lento; constata-se que, independentemente de disciplina e da proporção de alunas e país, elas abandonam as carreiras científicas em número muito superior aos homens em todas as etapas e especialmente após o doutorado (Rees, 2001).

De acordo com Jung \& Apedoe (2013), nos Estados Unidos a média de mulheres que cursam o Bacharelado em Ciência da Computação é menor do que 20\%, e a presença das mulheres nas carreiras relacionadas à Ciência da Computação só chega a $25 \%$. Estes autores também citam que uma das principais razões das meninas não escolherem carreiras relacionadas à Ciência da Computação é a falta de conhecimento sobre os diversos tópicos cobertos nos cursos superiores e sobre as diversas oportunidades oferecidas por estas carreiras. No século XXI ainda não se tem uma academia amistosa para as mulheres e persistem o clima frio e o teto de vidro (Cooper e Eddy et al, 2010), mesmo em países onde há legislação e políticas de combate à desigualdade (Kjeldala, Rindfleisha e Sheridana, 2005), sobretudo nos campos do conhecimento dominados por homens.

Nesse contexto, surgiu no Brasil o Programa Mulher e Ciência, em 2005, através de parceria entre vários ministérios (MCTI, CNPq, SPM, MEC, MDA) e a ONUMulheres, com três estratégias de intervenção: financiamento de projetos de pesquisa em gênero, mulheres e feminismos, instituição do Prêmio Construindo a Igualdade de Gênero para estudantes de ensino médio, graduação e pós-graduação, e realização do Encontro Nacional de Núcleos e Grupos de Pesquisa "Pensando Gênero e Ciências". Em 2013, foi lançada a chamada pública "Meninas e Jovens Fazendo Ciências Exatas, Engenharias e Computação" (CNPq/MCTI, SPM, Petrobrás), visando estimular a formação de mulheres para as carreiras de CTEM, articulando ensino médio (para atrair novas alunas) e ensino superior (para minimizar a evasão).

Entretanto, as políticas públicas para a inclusão feminina na Ciência e Tecnologia não têm tido a continuidade e a efetividade necessárias para garantir que as 
mulheres realmente ganhem novos espaços nesta grande área. Apresentamos a seguir dados e tendências de três cursos da área de Ciência da Computação construídos através da técnica de cenários que mostram a reduzida participação feminina neste espaço de atuação.

\section{Metodologia}

As tendências são baseadas em dados históricos sobre os quais são calculados os cenários futuros; relembra-se que toda a projeção é calculada isolando-se os fatores externos (que, caso ocorram, podem interferir nas projeções). Quando a análise é feita com dados históricos, o problema da desigualdade torna-se mais visível. Foram analisados os dados do alunado dos três cursos, por sexo, utilizando-se as seguintes variáveis preditivas: "entrada de graduandos" e "saída de graduados", em uma perspectiva longitudinal desde a fundação de cada curso, percorrendo-se o seguinte caminho metodológico:

(i) Transformação de números quantitativos em percentagem por sexo;

(ii) Cálculo do coeficiente de Pearson ( $\mathrm{R}>0,8$ em todos os conjuntos de dados, mostrando forte correlação) e da curva de tendência através da análise de regressão, técnica que permite explorar e inferir a relação de uma variável dependente com variáveis independentes, utilizando-se o método dos mínimos quadrados ordinários, adotando-se o modelo $\mathrm{Y}=\mathrm{a}+\mathrm{bX}$ (Triola, 2013);

(iii) Construção e interpretação dos gráficos contendo os dados em percentual e reta de tendência, possibilitando prospecção do cenário futuro provável.

Silva e Santos (2009) afirmam que apesar das inúmeras limitações dos modelos matemáticos da criação de cenários, eles possuem o mérito de, serem baseados no passado, apontarem o futuro, mesmo com a constatação de que o ambiente é mais complexo do que esses modelos podem prever. Esse é o caso desse artigo, onde todos os cálculos das curvas de tendência foram feitos através da análise de regressão utilizando dados históricos de cada curso da UFPB.

\section{Cenários e Tendências}

Vejamos o que se passou na IFES estudada nas últimas décadas, onde a pequena vantagem numérica das mulheres em matrículas gerais em 2017 nos cursos de graduação $(51,5 \%$ de 33.228$)(\mathrm{STI} / \mathrm{UFPB}, 2018)$ contrasta com a sua reduzida presença em cursos masculinos da área de Computação, Centro de Informática, foco do nosso estudo (Tabela 1).

Tabela 1: Discentes mulheres por curso do Centro de Informática, UFPB, 2017.

\begin{tabular}{|c|c|}
\hline Curso & Mulheres \\
\hline Ciência da Computação & $39(10,2 \%)$ \\
\hline Engenharia de Computação & $47(13,8 \%)$ \\
\hline Matemática Computacional & $38(21 \%)$ \\
\hline
\end{tabular}

Fonte: SIGAA (matrículas ativas em no período 2017.2) 
As projeções foram calculadas a partir do número de estudantes ingressantes a cada ano. Os gráficos mostram o percentual de homens e mulheres ingressantes e a curva de tendência calculada a partir destes percentuais.

\subsection{Ciência da Computação}

Quando este curso foi fundado, em 1985, atraiu um maior número de mulheres. Na primeira turma elas eram 36,4\% e chegaram a 40,7\% em 1993; entretanto, uma queda acentuada no ingresso feminino se deu a partir do ano 2000. A média de mulheres no período analisado é $21,3 \%$ e a de homens $78,7 \%$ (Figura 1). Há uma tendência de diminuição das mulheres a uma taxa de $0,4 \%$ aa, ou seja, a desigualdade aumenta. Sem ações afirmativas para reverter essa tendência, não haverá mais mulheres em 2050.

Figura 1: Desigualdade de sexo/gênero na Ciência da Computação

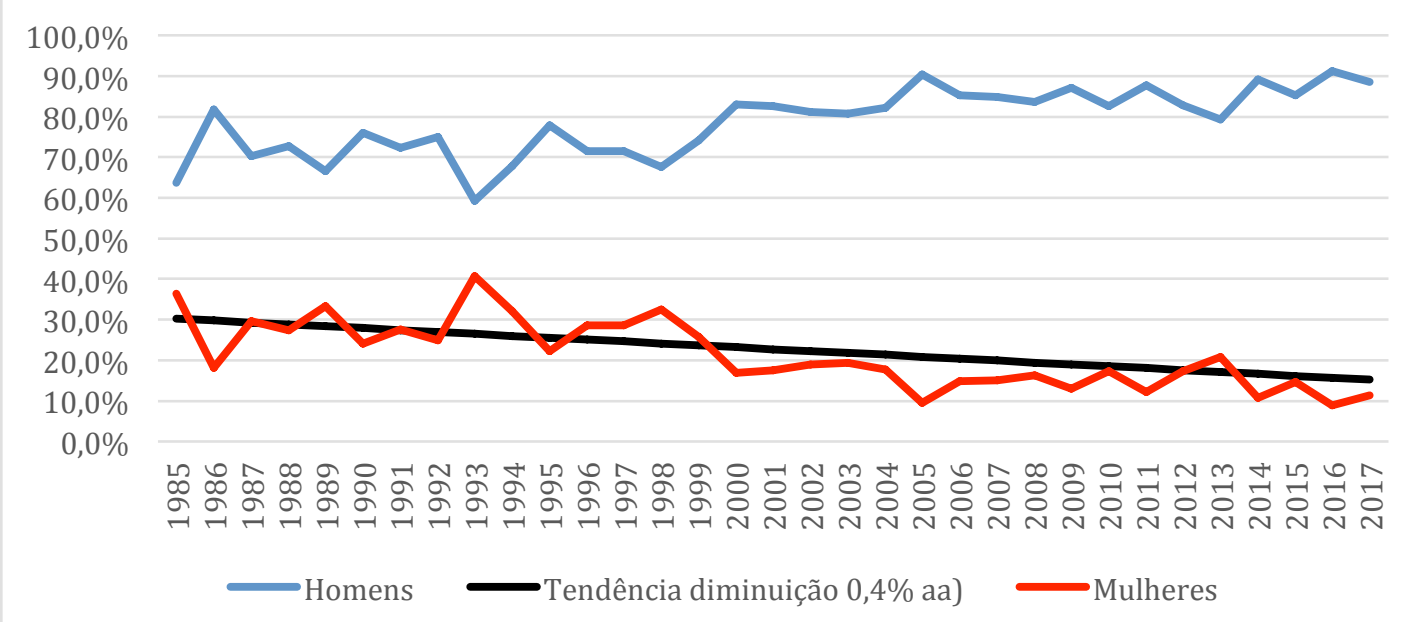

Fonte: Elaboração própria a partir de dados brutos fornecidos pelo STI/UFPB, 2018.

\subsection{Engenharia de Computação}

Neste curso, criado em 2011, o cenário futuro é de tendência de crescimento da defasagem entre homens e mulheres (Figura 2), pois há diminuição na entrada de mulheres a $0,7 \%$ aa. A média de homens é $87 \%$ e de mulheres de $13 \%$. Na ausência de ações afirmativas para modificar esse processo, no ano 2031 não haverá mais nenhuma mulher neste curso, na contramão da inclusão feminina. 
Gráfico 5: Desigualdade de sexo/gênero na Engenharia de Computação

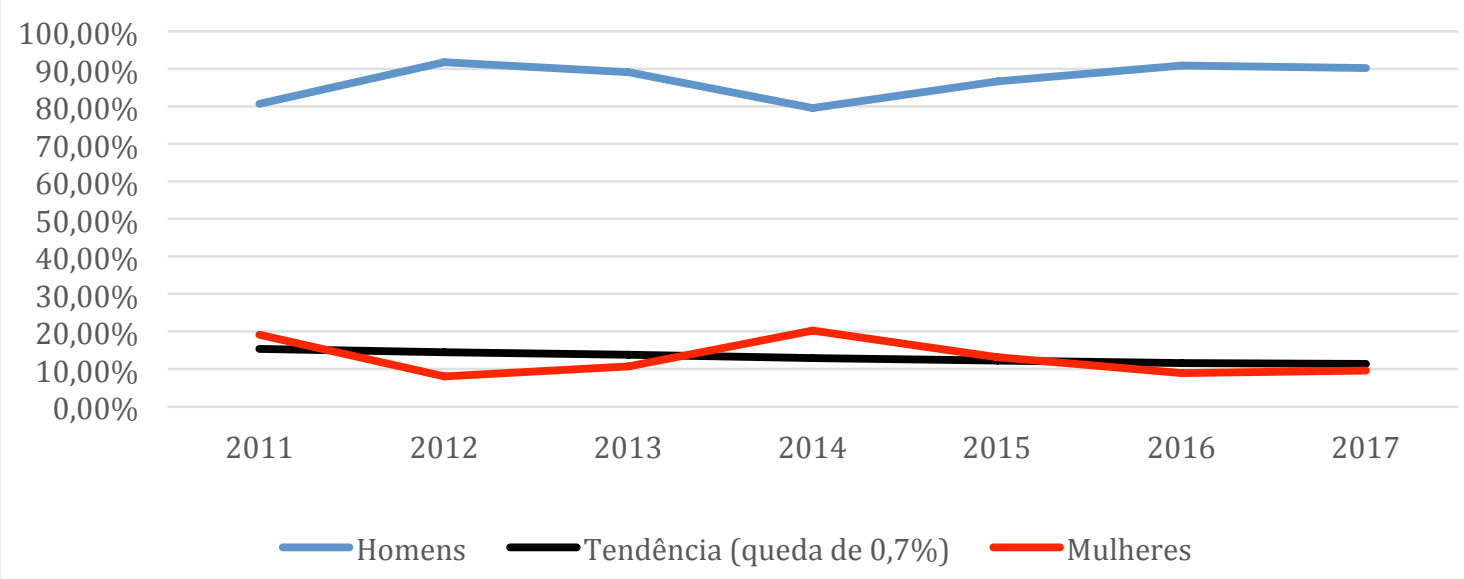

Fonte: Elaboração própria a partir de dados brutos fornecidos pelo STI/UFPB, 2018.

\subsection{Matemática Computacional}

Também recente (criado em 2012), este curso tem média de homens de 83,2\%, de mulheres de $16,8 \%$. Seu cenário é indicativo de estagnação, pois há uma tendência de diminuição das mulheres em $0,01 \%$ ao ano (Figura 3).

Figura 3: Desigualdade de sexo/gênero na Matemática Computacional

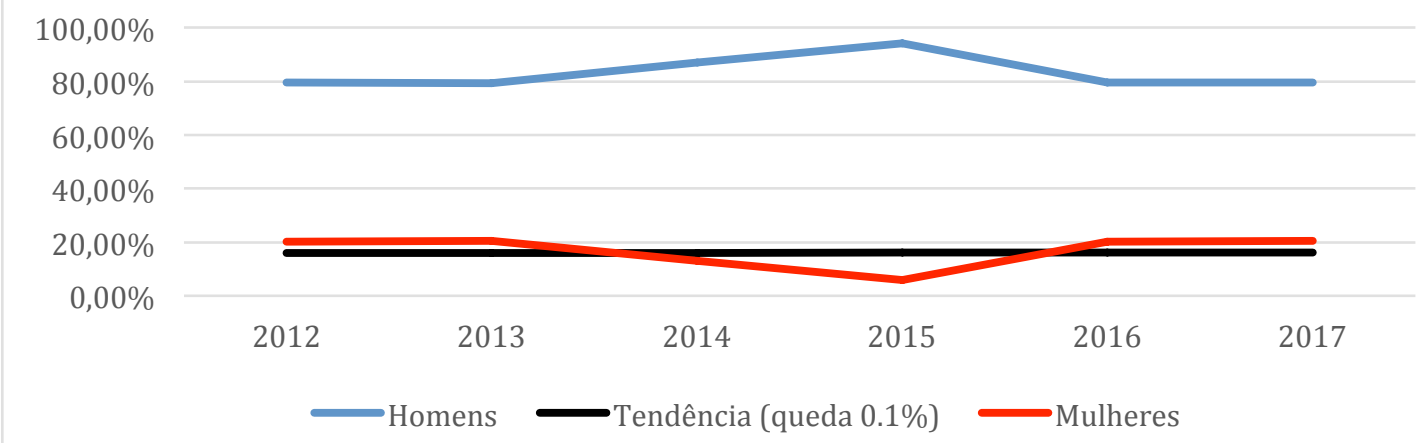

Fonte: Elaboração própria a partir de dados brutos fornecidos pelo STI/UFPB, 2018. 


\section{Comentários Finais}

Quando se considera a média de ingressantes em cada período analisado, o ingresso no último ano e a tendência projetada por sexo para cada curso, conforme os gráficos apresentados e a Tabela 2, constata-se que o cenário futuro segue na contramão da equidade de gênero proposta pelos Objetivos de Desenvolvimento Sustentável (ONU, 2015). Portanto, se não houverem ações que interfiram nestas tendências, dificilmente haverá igualdade de sexo/gênero na IFES pesquisada.

Tabela 2: Resumo das tendência de (des)igualdade de sexo/gênero em cursos da área de Computação, UFPB, 2017

\begin{tabular}{|c|c|c|c|c|c|}
\hline \multirow{2}{*}{$\begin{array}{c}\text { Cursos e período } \\
\text { analisado }\end{array}$} & \multicolumn{2}{|c|}{ Média no período } & \multicolumn{2}{|c|}{ Matrículas 2017.2 } & \multirow{2}{*}{ Tendência } \\
\cline { 2 - 5 } & Homens & Mulheres & Homens & Mulheres & \\
\hline $\begin{array}{c}\text { Ciência da } \\
\text { Computação } \\
32 \text { anos }\end{array}$ & $78,7 \%$ & $21,3 \%$ & $88,9 \%$ & $11,1 \%$ & $\begin{array}{c}\text { Aumento da desigualdade } \\
\text { a uma taxa } 0,4 \% \text { aa }\end{array}$ \\
\hline $\begin{array}{c}\text { Engenharia de } \\
\text { Computação } \\
7 \text { anos }\end{array}$ & $87,1 \%$ & $12,9 \%$ & $90,25 \%$ & $9,75 \%$ & $\begin{array}{c}\text { Aumento da desigualdade } \\
\text { a uma taxa de } 0,7 \% \text { aa }\end{array}$ \\
\hline $\begin{array}{c}\text { Matemática } \\
\text { Computacional } \\
7 \text { anos }\end{array}$ & $83,2 \%$ & $16,8 \%$ & $79,63 \%$ & $20,37 \%$ & $\begin{array}{c}\text { Estagnação (taxa de } 0,01 \% \\
\text { aa de aumento da } \\
\text { desigualdade) }\end{array}$ \\
\hline
\end{tabular}

Fonte: Elaboração própria a partir de dados brutos fornecidos pelo STI/UFPB, 2018.

Sabemos que a inclusão das mulheres em áreas da Ciência e Tecnologia não depende somente de políticas curriculares focadas na igualdade de gênero na educação básica e de programas de ação afirmativa que estimulem seu ingresso na educação superior, o que não se fez ainda no Brasil. Mais amplamente, é preciso mudar a organização patriarcal da família e do trabalho, através de "um entendimento crítico de gênero, de como ele funciona na ciência e na sociedade" (Schienbinger, 2001, p.39).

Nesse contexto, entendendo inclusão de gênero nas dimensões quantitativa e qualitativa, como políticas de paridade e transversalidade, consideramos que a universidade deveria assumir o protagonismo na realização dessas políticas, intra e extramuros. Uma das possibilidades de ação são os projetos de extensão junto às escolas da comunidade (Dos Santos, 2017; Mattos, 2015). Isso compreende informar, conscientizar e sensibilizar sobre o problema, especialmente os professores, sem esquecer que para as mulheres o primeiro passo para o empoderamento é tomar consciência dos preconceitos.

Hill, Corbett e St. Rose (2010) fazem várias recomendações inadiáveis nessa direção, principalmente se direcionadas ao ensino médio:

- divulgar as contribuições históricas e os sucessos atuais de mulheres em STEM e promover modelos femininos nas respectivas carreiras;

- atrair e reter mais alunas, cultivando ambientes acadêmicos que acolham, apoiem e reconheçam seus interesses e realizações, investindo em currículos que enfatizem aplicações concretas do conhecimento, e atentando para a influência de preconceitos e vieses (geralmente inconscientes) no ensino, orientação e avaliação; 
- atrair e reter docentes mulheres para a área de tecnologia, provendo mentoras, quando possível, para as alunas. Apesar de ser uma ação sobre o quadro docente, espera-se que impacte na atração de alunas para esta grande área.

A nossa recomendação é quanto à criação e adoção de políticas públicas que impactem nas escolhas de carreiras por parte das alunas do Ensino Médio, a fim de que estas sintam-se capazes, empoderadas e estimuladas a adentrar as profissões tipicamente masculinas da área de tecnologia. Consequentemente, poderá haver uma mudança nos números e nos cenários futuros de subáreas da tecnologia como a Ciência da Computação, objeto de estudo do nosso trabalho.

\section{Referências}

Blickenstaff, J. C. Women and science careers: leaky pipeline or gender filter? Gender and Education, 17 (4), 369-386. 2015.

Bourdieu, P. A Dominação Masculina. Rio de Janeiro: Bertrand Brasil, 1999.

BRASIL MCTI. Estratégia Nacional de Ciência, Tecnologia e Inovação 2016 - 2019. Brasília: Ministério da Ciência Tecnologia e Inovação (MCTI). 2016. Disponível em http://www.mcti.gov.br/documents/10179/1712401/Estrat\%C3\%A9gia+Nacional+de+C i\%C3\%AAncia\%2C\%20Tecnologia+e+Inova\%C3\%A7\%C3\%A3o+20162019/0cfb61e1-1b84-4323-b136-8c3a5f2a4bb7, acessado em Jan/2017.

BRASIL. (2013) Presidência da República. Secretaria Nacional de Políticas para as Mulheres. Plano Nacional de Políticas para as Mulheres. Brasília: Secretaria Nacional de Políticas para as Mulheres, 2013, 114 p. Disponível em http://www.compromissoeatitude.org.br/wpcontent/uploads/2012/08/SPM_PNPM_2013 .pdf, acessado em Abr/2015.

Carvalho, M. E, Silva, R. M., Aires, J. A. Estimulando o Ingresso de Meninas na Ciência e Tecnologia Através do Ensino de Computação. Encuentro Científíco Internacional del Comité FES de Sociología del Género, Valencia, 2018.

Cooper, J., Eddy, P., Hart, J., Lester, J., Lukas, S., Eudey, B., Glazer-Raymo, J., Madden, M. Improving gender equity in postsecondary education. In: S. S. Klein (Gen. Ed.). Handbook for Achieving Gender Equity through Education (2 ed., pp. 631-653). New York and London: Routledge. 2010.

Dos Santos, J. M. O., Souza, C. M., Santos, T. A., Alves, P. M. B. F., \& Santos, D. A. Contribuições da Extensão Universitária na formação social, acadêmica e profissional dos estudantes de Computação. Em XXXVII CSBC, 25ำ Workshop de Educação em Computação (WEI-2017), São Paulo, 2017.

Hill, C., Corbett, C. e St. Rose, A. Why so few? Women in Science, Technology, Engineering, and Mathematics. AAUW, Washington DC. 2010.

JUNG, Eunjin, APEDOE, Xornam. Changing Young Women's Perceptions of CS via Outreach. ITiCSE'13, the 18th Annual Conference on Innovation and Technology in Computer Science Education, Inglaterra. 2013. 
Kjeldala, S. E., Rindfleisha, J. e Sheridana, A. Deal making and rule breaking: behind the façade of equity in academia. Gender and Education, 17 (4), p. 431-447. 2005.

Lima, B. S. e Costa, M. C. da. Gênero, ciências e tecnologias: caminhos percorridos e novos desafios. Cadernos Pagu, Campinas, n. 48. 2016. Disponível em http://www.scielo.br/scielo.php?script=sci_arttext\&pid=S0104$83332016000300304 \& \operatorname{lng}=$ pt\&nrm=iso, acessado em Fev/2018.

Mattos, G. O, Silva, D. Moreira, J.A. A Utilização de Kits de Robótica como Ferramenta para o Ensino de Programação à Meninas do Ensino Médio. Em XXXV Congresso da Sociedade Brasileira de Computação, 23o Workshop de Educação em Computação (WEI-2015), Recife, 2015.

ONU. Gender mainstreaming. An overview. New York: Office of the Special Adviser on Gender Issues and Advancement of Women. Department of Economic and Social Affair, 2002. http://www.un.org/womenwatch/osagi/pdf/e65237.pdf. Acessado em 01/03/2018.

ONU. Objetivos de Desenvolvimento Sustentável - ODS Agenda 2030. 2015. http://www.onumulheres.org.br/planeta5050/. Acessado em 21/02/2018.

Rees, T. (2001). Mainstreaming Gender Equality in Science in the European Union: The 'ETAN Report'. Gender and Education, 13(3), 243-260.

Schienbinger, L. (2001). O feminismo mudou a ciência? (R. Fiker, Trad.). Bauru, SP: EDUSC.

Schwartz, Peter. A arte da visão de longo prazo: planejando o futuro em um mundo de incertezas. São Paulo: Best Seller, 2000.

Silva, Ricardo Moreira da; SANTOS, João Luiz Fonseca. A Incorporação da Imaterialidade Humana na Construção de Cenários. XXIX Encontro Nacional de Engenharia de Produção. Salvador, BA, Brasil, 06 a 09 de outubro de 2009.

STI/UFPB. Superintendência de Tecnologia da Informação da Universidade Federal da Paraíba. 2018.

Triola, Mario F. Introdução A Estatística - Atualização da Tecnologia - $11^{\mathrm{a}}$ Ed. Editora LTC. 2013. 